\title{
cTRAIN: A computer-aided training system developed in SuperCard for teaching skills using behavioral education principles
}

\author{
W. KENT ANGER and DIANE S. ROHLMAN \\ Oregon Health Sciences University, Portland, Oregon \\ JOHN KIRKPATRICK \\ Painters District Council No. 5, Portland, Oregon \\ RONALD R. REED \\ Ronald R. Reed Consultants, Portland, Oregon \\ CHRISTINE A. LUNDEEN \\ Oregon Health Sciences University, Portland, Oregon \\ and \\ DAVID A. ECKERMAN \\ University of North Carolina, Chapel Hill, North Carolina
}

\begin{abstract}
A computer-aided training program was developed in SuperCard and piloted with professional painters. Taking a modern programmed-instruction/behavioral-education approach, cTRAIN is structured as a series of information sets. Each information set consists of a series of information screens (three to five recommended) followed by quiz screens (one to three recommended) structured as fourresponse multiple choice questions. Correct quiz responses produce positive feedback and continuation in the series, whereas incorrect responses result in "error" feedback and return the student to the beginning of the information set to repeat the same information screens and the same quiz question. This report demonstrates a specific implementation, respiratory protection requirements, using the flexible cTRAIN system for developing training modules. Fifteen adults completed the respiratory protection program, demonstrating substantial and significant $(p<.0001$ by paired $t$ test) gains from baseline pretest (19.4 out of 30 questions) to the immediate posttest (28.1). Performance remained elevated (26.4) on a retest taken 1 week later.
\end{abstract}

Training, as distinct from academic education, is the term applied to the teaching of skills, often to working populations. Indeed, United States corporations spend between $\$ 55$ and $\$ 60$ billion per year to train an estimated 60 million employees (National Institute for Occupational Safety and Health, 1999), including training in safe workplace behavior. There is empirical (e.g., Merrill, Reiser, Merrill, \& Landes, 1995) and consensus agreement that individual tutorials can be the most effective approach to teaching knowledge and changing behavior in working populations (Cohen \& Colligan, 1998). How-

Funding for the development of the cTRAIN program was provided by the Center for Research on Occupational and Environmental Toxicology at Oregon Health Sciences University (Portland) and Oregon OSHA (Salem). The manuscript was prepared with funding from NIOSH (1 R01 OH04230-01 and R01 OH04193-01). The cTRAIN program is copyrighted by Oregon Health Sciences University and Ronald R. Reed Consultants, Inc. Correspondence concerning this article should be directed to W. K. Anger, Oregon Health Sciences University, 3181 SW Sam Jackson Park Road L606, Portland, OR 97201 (e-mail: anger@ ohsu.edu). ever, occupational specialists have few tools to produce effective and customized training.

As a tool to fill this gap, we have developed cTRAIN, a computer-based training program, founded on proven behavioral training principles, that ensures knowledge competency at completion and can be readily revised as requirements change. The behavioral or behavior-analytic training principles employed have gained dominance for training basic intellectual and life skills to individuals who are slow to learn (see, e.g., Journal of Applied Behavioral Analysis, 1968-present). They were selected because they ensure effectiveness in training people with limited educational and study skills, who may be represented in blue collar occupations, and because they are effective principles for teaching content to any population. The principles that underlie this approach, are the following: (1) fully specify learning objectives and focus attention on key elements, (2) carefully sequence training steps to ensure competence at each point, (3) adopt learner-paced progress through training steps, and (4) provide a precise record of accomplishments (see, e.g., Edgar \& Sulzbacher, 
1992). More specifically, the model for the cTRAIN program is programmed instruction (Holland, 1960, 1967; Skinner, 1958). Our goal was to develop a computerbased training program that could be employed to teach essential skills and information, such as health and safety, to a broad range of workers in a cost-effective manner.

\section{ELEMENTS OF THE cTRAIN PROGRAM}

IncWell's SuperCard program was selected as the development environment for cTRAIN because (1) it is a mature and widely used programming environment for developing multimedia programs, (2) SuperCard programs can link to the Macintosh operating system's spoken text capability to produce on-demand computer-generated English and Spanish speech that "speaks" the text typed into the content program, and (3) SuperCard programs can be distributed without cost as runtime programs. Presentation requires a Macintosh computer with a mini- mum 12.1-in. screen (thus being suitable for recent PowerBooks and iBooks) and Mac OS 7.0 or higher; a color or gray scale screen is required.

The cTRAIN program is divided into a Screenbuilder environment in which a "content specialist" enters information (including pictures or movies) and quiz questions, and a standalone presentation program that "instructors" initiate for the "students" to complete at their own pace. The underlying assumption is that the students may have minimal education or literacy and be unfamiliar with computers, but the program must also be acceptable to and suitable for college-educated students.

\section{cTRAIN Screenbuilder Environment}

The Screenbuilder environment allows content specialists with no programming knowledge to enter information and pictures to create a new training program. The cTRAIN program consists of information screens (Figure 1) and quiz screens (Figure 2).

\section{Respiratory Protection}

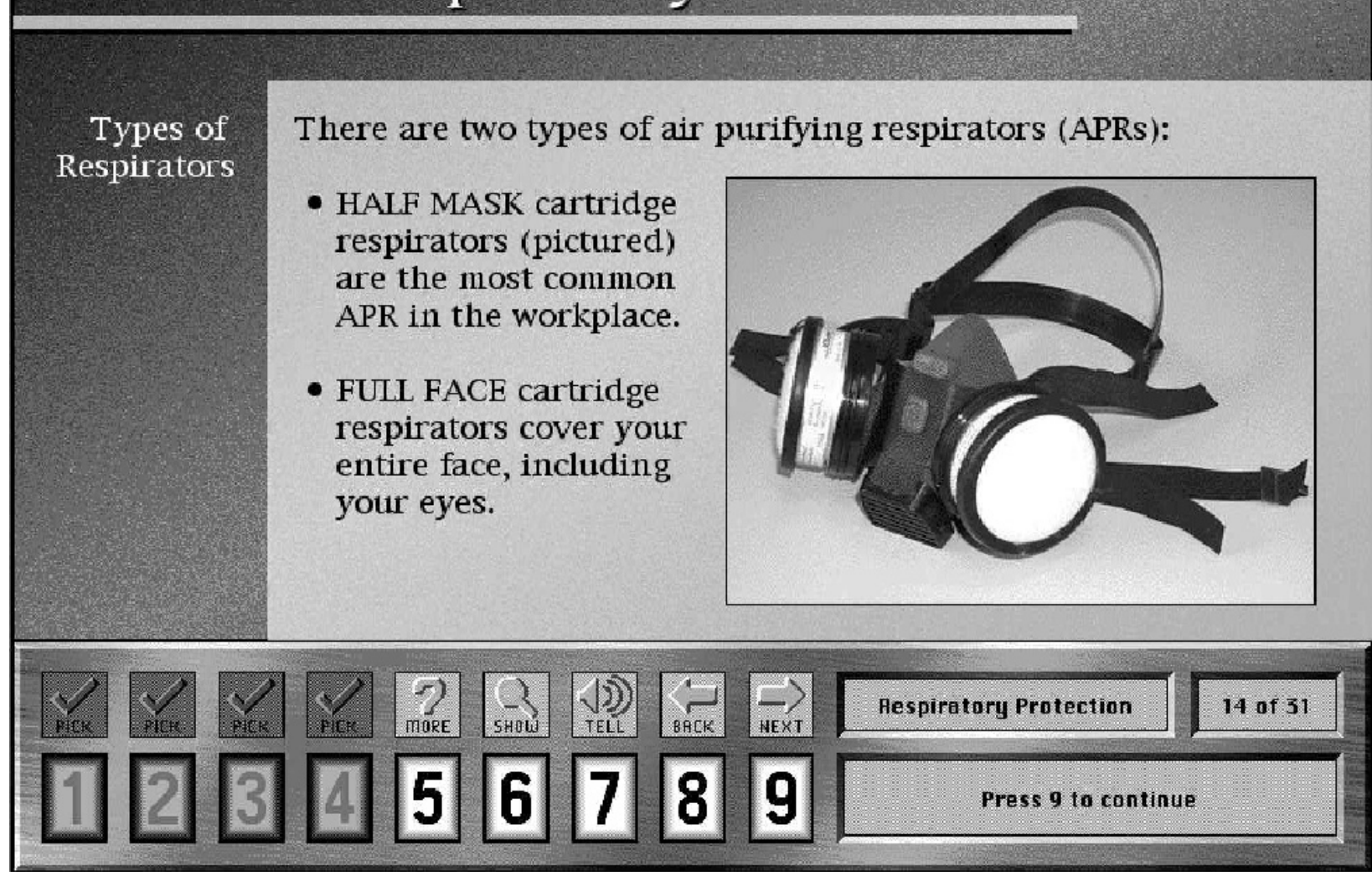

Figure 1. Typical information screen from the Respiratory Protection demonstration. In the navigation bar at the bottom of the figure, the brighter icons and black numbers with a light surround indicate actions the student can take ("more" is to view screens with nonrequired information that will not be on the quizzes, "show" is to enlarge a picture or play a QuickTime movie, "tell" is to hear the screen text spoken, "back" is to make a transition to preceding information screens, and "next" is to make a transition to the next screen in the series). To the right on the navigation bar is the program topic, the current location in the series (screen 14 of 31 total screens), and the most probable action to take (press 9 to continue to the next screen). The figure is in gray-tone; the screen appears in color on color monitors. 


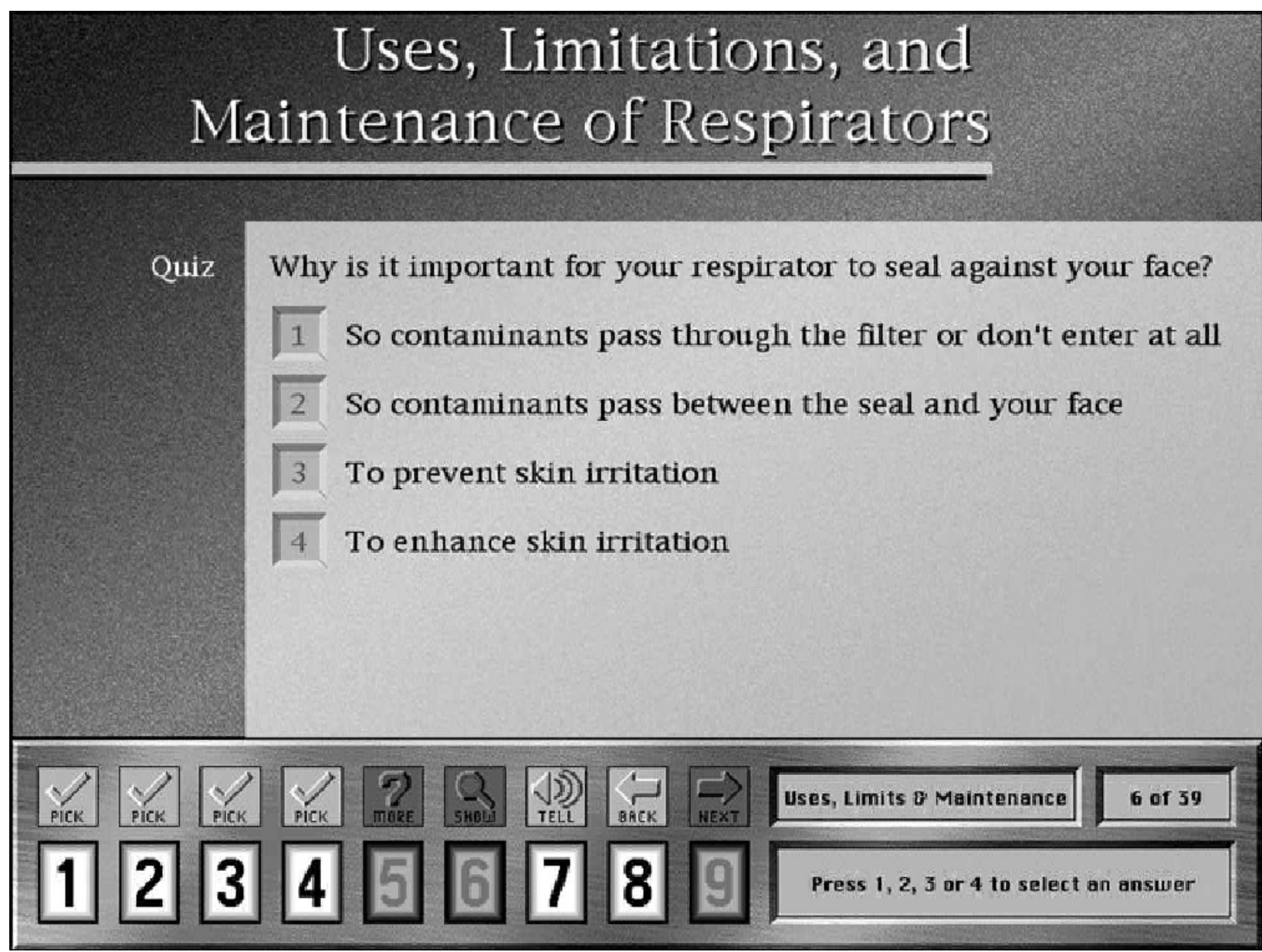

Figure 2. Typical quiz screen from the Respiratory Protection demonstration. In the navigation bar at the bottom of the figure, the brighter icons and black numbers with a light surround indicate actions the student can take ("pick" is for choosing an answer, "tell" is to hear the screen text spoken, "back" is to make a transition to preceding information screens to review information before selecting an answer). To the right on the navigation bar is the specific topic, the current location in the series (screen 6 of 39 total screens), and the most probable action to take (select an answer). The figure is in gray-tone; the screen appears in color on color monitors.

Information screens (Figure 1) are divided into four main areas: (1) the title at the top of the screen, (2) the subtitle on the left side, (3) the information area in the center, and (4) the navigation bar on the bottom of the screen. The content specialist can (1) enter the main title and subtitle, (2) enter text (of any font or size) and one pictorial element (digital picture or QuickTime movie) in the information area, (3) enter a suggested action in the navigation bar, (4) select from among several colors for the screen background and navigation bar, and (5) substitute natural spoken language digitized recordings for the computer-generated speech (thus allowing any language).

Quiz screens (Figure 2) are identical to information screens, except that the information area is replaced by a prestructured multiple choice question (into which pictorial elements can be inserted) with two, three, or four answers.

The cTRAIN program enforces a grouping structure termed information set, which consists of one or more in- formation screens (three to five recommended) followed by one or more quiz screens (one to three recommended). An information set, in the presentation program described next, becomes a self-contained sequence through which the student must progress serially, continuing through all the information screens followed by all the quiz screens. Errors on quiz screens disrupt this flow, returning the student to the first information screen in the information set. There are no practical limits on the number of information screens or quiz screens in an information set or in the number of information sets in the program. If the content specialist is creating both Spanish and English versions of the training, two separate programs must be created, one in each language.

To create a program in cTRAIN Screenbuilder, the content specialist "builds" a series of information sets. The number of information screens and quiz screens is selected for each new information set, after which the new information set (with blank screens ready for con- 
tent to be entered into) is placed into the series of information sets at any location in the series. The content specialist then enters text and pictures into the information screens and questions into the quiz screens. At the time the information set is "built," the quiz screens are duplicated to create a test that is presented at the end of the completed series of information sets (the posttest) and can optionally be presented as a pretest prior to beginning training. It is possible to scramble the order of questions in the posttest (and in the identical pretest) or leave them in the same order in which they appear in the information set series.

\section{Presentation Program}

The presentation program is a separate, standalone runtime program for delivering the content entered into cTRAIN Screenbuilder, as a student-friendly training activity. When setting up the presentation program, the instructor can elect to present (or accept defaults for) student user instructions, the pretest for establishing the baseline knowledge level of the student, the language (English or Spanish), the operating system voice (from the Mac OS options), the data storage location, and "pass percentages" for a report of pre- and posttest performance.

From the perspective of the student, the presentation program begins with optional information screens that describe how to use the program and with an optional pretest (which is identical to the posttest). The program may be divided into topics, from which the student selects at the outset of training, in order to allow a large amount of information to be separated into smaller units of appropriate length for the intended audience (this action must have been taken in cTRAIN's Screenbuilder). The student progresses serially through each information set at his or her own pace. The exception to this is that an error on a quiz screen returns the student to the beginning of the information set, whereas correct completion of a quiz screen conveys the student to the next quiz screen or, if it is the final quiz screen in the set, to the next information set in the program. Stylized smiling or frowning faces and text indicate the success of each quiz question choice, serving as feedback. It is not possible to return to an information set once the student has exited into the next information set. The posttest occurs after the student completes the last information set. If the information sets are divided into topics, the test on each topic occurs at the end of the topic. The frequent quizzes, feedback, and self-pacing are the primary procedures that define this program as programmed instruction (see, e.g., Emurian, Hu, Wang, \& Durham, 2000; Holland, 1960; Skinner, 1958).

A key goal was that students should be able to learn from and navigate the program without assistance from an instructor. The primary method for accomplishing this is the navigation bar that appears at the bottom of every screen (Figures 1 and 2). The bar highlights actions that are currently available to the student by surrounding the stylized buttons and illustrative icons in a light color (they are grayed out if not available, as in Figures 1 and 2 ). The student's options are to see nonrequired content by pressing the " 5 " button, see a movie by pressing " 6 ," listen to text spoken by pressing " 7 " (note that this is a screen-by-screen student-initiated option), and step back to earlier screens in an information set by pressing "8." The current screen and the total number of screens are on the right, to provide an indicator of how many screens remain to be completed. Complementing the training program is a durable "9BUTTON" response input unit (see, e.g., Anger et al., 1996) that is readily used by less educated users who shy away from computer keyboards (e.g., Rohlman et al., 2000), as well as a keyboard option. Data are saved, and a printable performance report is generated.

\section{DEMONSTRATION}

Occupational Safety and Health Administration(OSHA) requirements for respirator use provided the content for the first cTRAIN program. Prior to undertaking a job requiring respiratory protection, workers are required to know basic information, which we divided into three topics: (1) uses, limitations, and maintenance of respirators, (2) selection of proper respiratory protection, and (3) recognition of respiratory hazards. The program consisted of 45 information screens and 30 four-answer multiple choice quiz screens distributed among 14 information sets. The pre- and posttests consisted of the same 30 quiz screens as those presented in the training program.

Fifteen painters and dry wall finishers ( 2 females, 13 males) provided feedback, leading to changes in the structure of the program. Comments on the computergenerated speech were generally neutral, but more important, all 15 respondents rated it as understandable, a key factor in the economy of this method.

\section{Effectiveness in Teaching Respiratory Protection}

Fifteen participants recruited by newspaper advertisement were paid $\$ 80$ for completing the training and repeating the test 1 week later. Following informed consent, the participants ( 8 females, 7 males; mean age, 35.3 years; mean education, 15.2 years) completed the Respiratory Protection program on Macintosh G3 Powerbooks with active matrix color screens using a 9BUTTON response unit.

Performance improved significantly $(p<.0001$ by paired $t$ test) from pretest $(19.4[S D=3.8]$ correct of 30 possible) to posttest $(28.1$ [ $S D=2.9]$ correct $)$. Retention after 1 week was 26.4 correct $(S D=3.5)$. All 15 participants navigated the program successfully. None asked questions about how to navigate or use the program.

In summary, cTRAIN is in an effective, modern programmed instruction/behavioral education approach for teaching health and safety to adults in the workforce. This fills an important gap in skills training and has promise for a wider audience. Its utility for assessing behavioral education principles in populations rarely studied could add to the generalization of those principles to a large and important population segment. 


\section{REFERENCES}

Anger, W. K., Rohlman, D. S., Sizemore, O. J., Kovera, C. A., GibERTINI, M., \& GER, J. (1996). Human behavioral assessment in neurotoxicology: Producing appropriate test performance with written and shaping instructions. Neurotoxicology \& Teratology, 18, 371-379.

Cohen, A., \& Colligan, M. J. (1998). Assessing occupational safety and health training (DHHS [NIOSH] Pub. No. 98-145). Washington, DC: U.S. Government Printing Off ice.

EdGar, G., \& Sulzbacher,S. (1992). Influences and effects of the behavioral paradigm in special education. In R. P. West \& L. A. Mamerlynck (Eds.), Designs for excellence in education: The legacy of B. F. Skinner (pp. 187-217). Longmont, CO: Sopris West.

Emurian, H. H., Hu, X., Wang, J., \& Durham, A. G. (2000). Learning Java: A programmed instruction approach using Applets. Computers in Human Behavior, 16, 395-422.

Holland, J. G. (1960). Teaching machines: An application of princi- ples from the laboratory. Journal of the Experimental Analysis of Behavior, 3, 275-287.

Holland, J. G. (1967). A quantitative measure for programmed instruction. American Educational Research Journal, 4, 87-101.

Merrill, D. C., Reiser, B. J., Merrill, S. K., \& Landes, S. (1995). Tutoring: Guided learning by doing. Cognition \& Instruction, 13, 315-372.

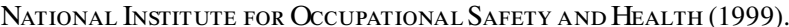

TIER: A model for research on training effectiveness (DHHS [NIOSH] Pub. No. 99-142). Washington, DC: U.S. Government Printing Office.

Rohlman, D. S., Bailey, S. R., Brown, M., Blanock, M., Anger, W. K., \& McCAuley, L. (2000). Establishing stable test performance in tests from the Behavioral Assessment and Research System (BARS). NeuroToxicology, 21, 715-724.

SKINNER, B. F. (1958). Teaching machines. Science, 12, 969-977.

(Manuscript received November 8, 2000; revision accepted for publication March 11, 2001.) 